\title{
Optimized CMOS Design of Full Adder using 45nm Technology
}

\author{
Sheenu Rana \\ M.E.Scholar \\ Departmant of Electronics \& \\ Communication Engineering \\ NITTTR, Chandigarh, India
}

\author{
Rajesh Mehra \\ Associate Professor \\ Departmant of Electronics \& \\ Communication Engineering
}

\begin{abstract}
This paper presents low power full adder designed with pass transistor logic which reduces the area, power and delay. we compared conventional 28T CMOS full adder with 16T and 8T full adder in terms of area, power and delay using 45um Technology

The schematic of all three design has been developed and its layout has been created using micro-wind tool. The result show that $8 \mathrm{~T}$ full adder consumes $98 \%$ less power as conventional $28 \mathrm{~T} \& 65 \%$ less power compared to $16 \mathrm{~T}$ full adder.
\end{abstract}

\section{Keywords}

Full adder, Pass Transistor logic(PTL), Transmission gate (TG) ,CMOS, Drive current, Channel Length.

\section{INTRODUCTION}

Power consumption is an important efficiency factor in designing Very Large Scale Integrated (VLSI) Circuit. Moreover, with the explosive growth of VLSI technology the demand and popularity of portable devices has driving designers to strive for smaller silicon area, higher speeds, longer battery life, less power consumption and more reliability electronics circuits. The design criterion of a full adder cell involves transistor counts which largely affects the design complexity of many function units such as multiplier and algorithmic logic unit digital adder

Full adder is made up of two half adders and or gate.

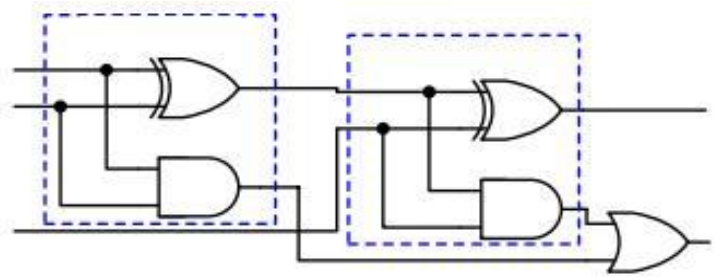

Fig1: circuit diagram of full adder Circuit has three inputs A ,B and $C$ and two outputs sum and carry. Circuit must satisfy the truth table given below.

(ALU)[1,2] The speed of the design is limited by size of the transistors, parasitic capacitance and delay in the critical path The driving capability of a full adder is very important as they are mostly used in cascade configuration, where the output of one provides the input for other. Several full adder circuits have been proposed targeting on design accents such as power, delay and area. Among those designs with less transistor count using pass transistor logic have been widely used to reduce power consumption $[3,4]$.These designs can be divided into two types, the CMOS logic and the pass -transistor logic [5]. In this paper, we have given a brief description of the evolution of full adder circuits in terms of lesser power consumption, higher speed and lesser chip size.

\subsection{Full Adder}

An adder is a digital electronic circuit that is used to perform addition binary numbers. Full adder is a combination circuit that performs addition of three bits

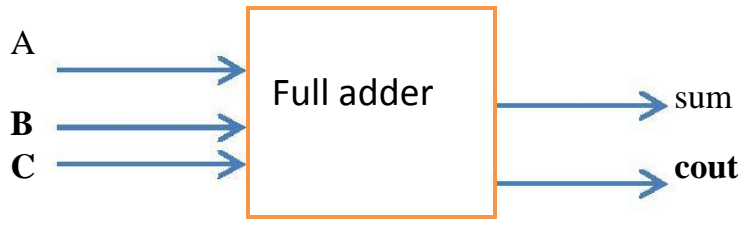

\begin{tabular}{|l|l|l|l|l|}
\hline A & B & Cin & SUM & Cout \\
\hline 0 & 0 & 0 & 0 & 0 \\
\hline 0 & 0 & 1 & 1 & 0 \\
\hline 0 & 1 & 0 & 1 & 0 \\
\hline 0 & 1 & 1 & 0 & 1 \\
\hline 1 & 0 & 0 & 1 & 0 \\
\hline 1 & 0 & 1 & 0 & 1 \\
\hline 1 & 1 & 0 & 0 & 1 \\
\hline 1 & 1 & 1 & 1 & 1 \\
\hline
\end{tabular}

Fig2:Truth table of full adder

\subsubsection{Pass Transistor Logic}

Pass transistors are single FETs that pass the signal between drain and source terminals instead of a fixed power supply value. The basic difference of pass - transistor logic compared to the CMOS logic style is that the source side of the logic transistor networks is connected to some input signals instead of the power lines. The advantage is that one pass -transistor network (either NMOS or PMOS) is sufficient to perform the logic operation, which results in a smaller number of transistors and smaller input loads.

\subsubsection{Transmission Gate}

An electrical feature of the transmission gate and pass transistor is that there are no direct signal connection to the power supply Vdd or ground . No isolation between the input and output.Output progressively deteriorates as it passes through various stages.

\section{COMPARATIVE ANALYSIS OF FULL ADDER}

\subsection{Conventional 28t Cmos Full Adder:}

A classical design of standard static CMOS full adder is based on regular CMOS structure with conventional pull-up and pulldown transistor providing full-swing output and good driving capabilities. The existence of the PMOS block in static CMOS circuits is a main drawback because it has low mobility 
compared to the NMOS. Hence, there is a need to be sized up to get desired performance.

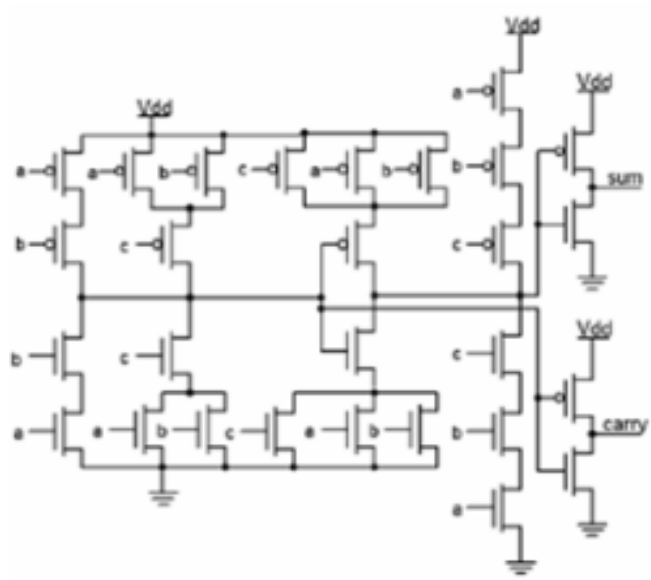

Fig3(A).Schematic of $28 \mathrm{~T}$ full adder

\subsection{6t Full Adder}

16-T full adder is shown in Figure(4B) below. The circuit consists of low power XOR and XNOR gates, pass transistors and transmission gates. The adder offers higher speed and lower power consumption than other implementations of the full adder.

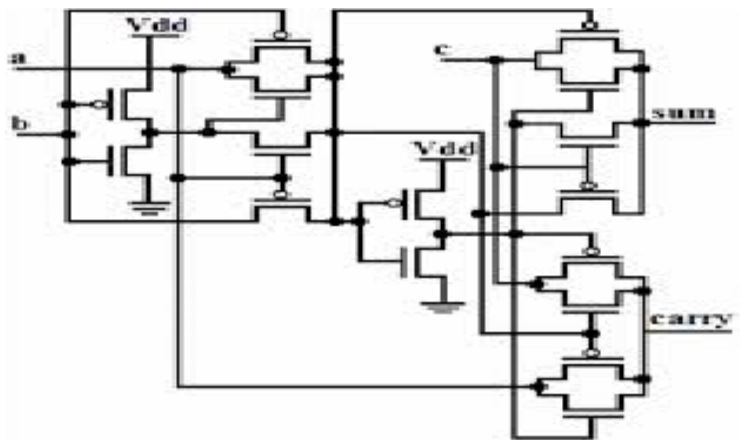

Fig: 3(b) schematic of $16 t$ full adder

\section{$2.38 t$ Full Adder}

The 8T full adder contains a two 3T PTL XOR gate, shown in Figure 4(c), two transmission gates based multiplexer designs for sum and Cout signals.

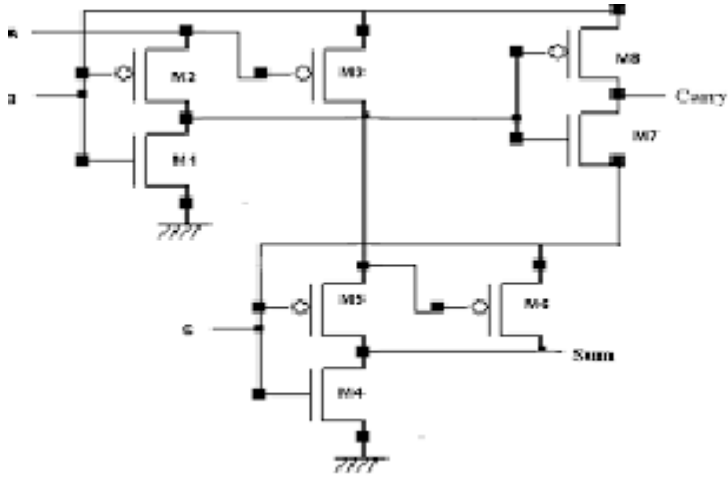

Fig 3(c) schematic of $8 \mathrm{~T}$ full adder.

\section{ADDER SCHEMATIC AND WAVEFORM.}

Logic level layout of 28Transistor conventional full adder circuit after schematic.

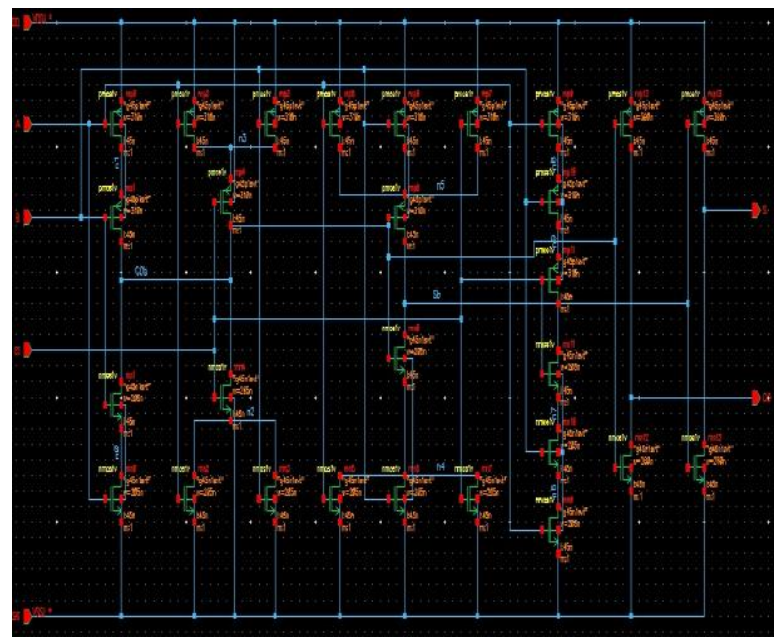

Fig 4(a) Schematic of 28t full adder

Voltage versus time graph after layout gives us the power consumed and the delay between the output and input.

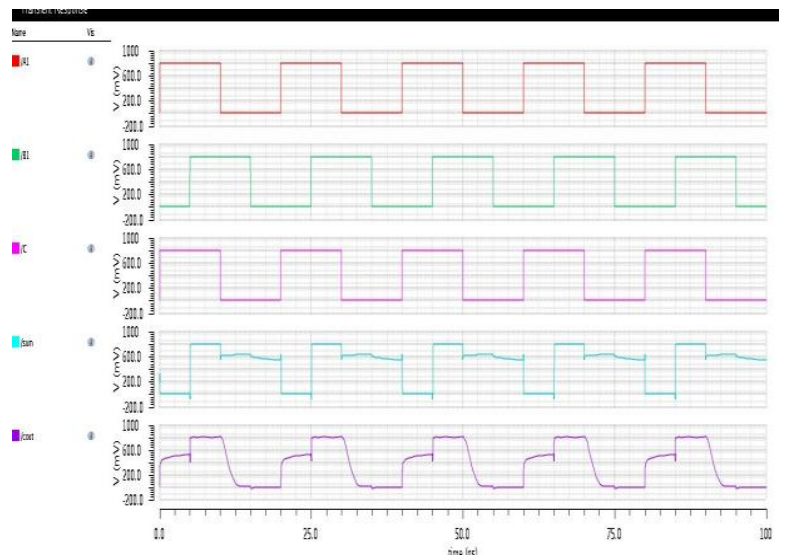

Fig 4(b) Voltage v/s Time graph

\subsection{T Full Adder}

Logic level layout of $16 \mathrm{~T}$ transistor full adder circuit schematic layout is shown in fig. 4(c) .

The circuit consists of low power XOR and XNOR gates, pass transistors and transmission gates.

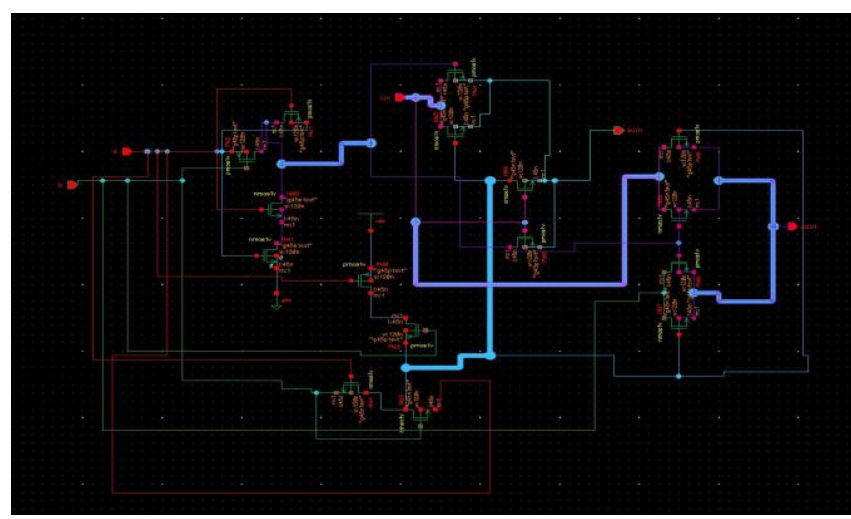

Fig 4( c) schematic of $16 \mathrm{~T}$ full adder 
Logic level layout of 16Transistor full adder using PTL.Reduced circuit layout below in fig 4(c) and voltage versus time graph of the layout is shown in figure $4(d)$.

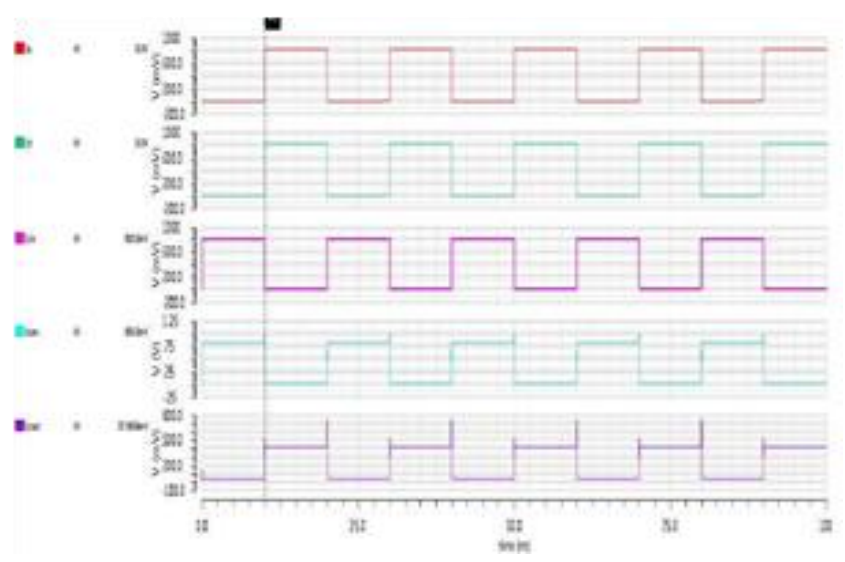

Fig 4(d) voltage v/s time graph.

\subsection{T Full Adder}

Logic level layout of 8Transistor full adder circuits schematic layout as shown

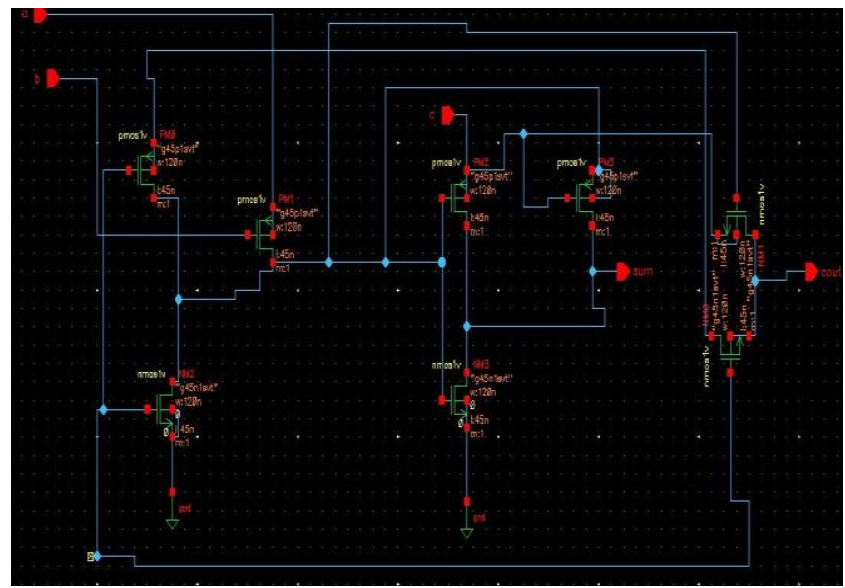

Fig 4(f) schematic of $8 \mathrm{~T}$ full adder

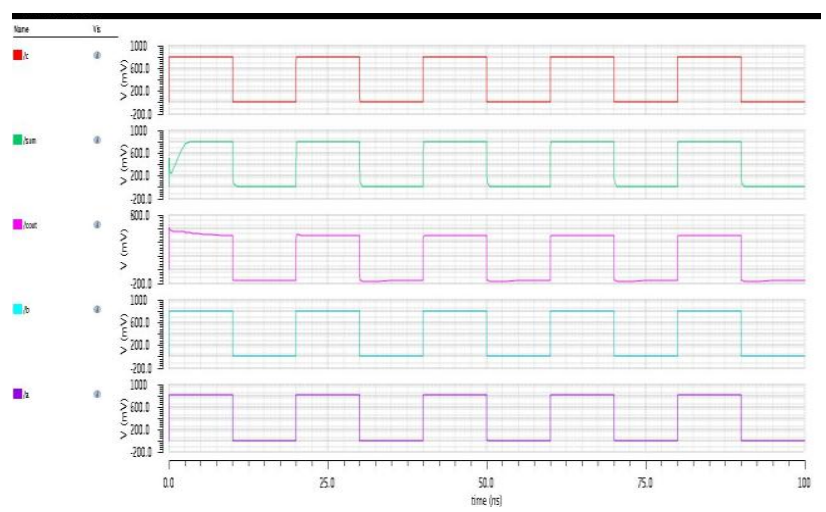

Fig $4(\mathrm{~g})$ voltage v/s time graph

\section{RESULT \& ANALYSIS}

Comparative analysis between conventional type,16T and 8T Full Adder is shown in Table I .The total no of transistor involved in the circuit, average power, delay and area are lowest for $8 \mathrm{~T}$ full adder
Table 1: Comaparitive Analysis

\begin{tabular}{|l|l|l|l|}
\hline PARAMETERS & CCMOS & $16 \mathrm{~T}$ & $8 \mathrm{~T}$ \\
\hline $\begin{array}{l}\text { No. of } \\
\text { transistors }\end{array}$ & 28 & 16 & 8 \\
\hline Power & $47.47 \mathrm{uw}$ & $20.7 \mathrm{uW}$ & $0.099 \mathrm{uW}$ \\
\hline Delay & $76 \mathrm{ps}$ & $65 \mathrm{ps}$ & $38 \mathrm{ps}$ \\
\hline Width & $42 \mathrm{um}$ & $25 \mathrm{um}$ & $13 \mathrm{um}$ \\
\hline
\end{tabular}

\section{CONCLUSION}

The PTL 8T circuit was designed with a 45um technology and were simulated and compared with other conventional and 16T full adder cell. Simulation results shows that average power consumption is less than both conventional and 16t full adder. Due to low power consumption, delay and less transistor count the $8 \mathrm{~T}$ full adder cell can be useful in portable and low power application.

\section{REFERENCES}

[1] Keivan Navi and Omid Kavehei , "Low-Power and HighPerformance 1-Bit CMOS Full-Adder Cell", JOURNAL OF COMPUTERS, VOL. 3, NO. 2, Feburary 2008,pp48-54

[2] Nidhi Tiwari, Ruchi shrma ,'Implementation of area and Energy efficient Full adder cell”, ICRAIE-2014,,May 0911,2014,Jaipur,978-983.

[3] Yi Wei, Ji-zhong Shen, "Design of a novel low power 8transistor I-bitfull adder cell", Journal of Zhejiang University SCIENCE C, July2011, Volume 12, Issue 7, pp 604-607

[4] Y. Jiang, AI-Sheraidah. A, Y. Wang, Sha. E, and J. Chung, "A novelmultiplexer-based low-power full adder," IEEE Trans. Circuits Syst. n,Analog Digit. Signal Process., July 2004, vol. 51, pp.345-348.

[5] Dan Wang, Maofeng Yang, Wu Cheng XUguang Guan, Zhangming Zhu,Yintang Yang " Novel Low power Full Adder Cells in 180nm CMOS Technology", 4th IEEE conference on Industrial Electronics and Applications, 2009,pp. 430-433.

[6] R. Zimmermann and W. Fichter, "Low -power logic styles: CMOS versus pass -transistor logic, || IEEE J. Solid-State Circuits, Vol. 32, July 1997,pp.1079-90

[7] Tanu sharma and Rajesh mehra,"Full adder design analysis for different logic styles on $45 \mathrm{~nm}$ channel length",International journal of computer technology ." 2014, ,pp95-99.

[8] A sharma ,R singh, Rajesh mehra,"Low power TG full adder using CMOS Technology",IEEE Parallel distributed and Grid Cmputing (PDGC) ." 2012

[9] A sharma ,Rajesh mehra,"Area and power efficient cos adder design by hybridizing PTL and GDI Technique",International journal of computer application" 2013,pp15-22.

\section{AUTHOR PROFILE}

Ms. Sheenu Rana: Ms. Sheenu Rana is currently associated with ARYANS College of Engineering and Technology, Nepra, Punjab, India since 2014. She is currently pursuing M.E from 
National Institute of Technical Teachers Training and Research, Chandigarh India. She has completed her B. Tech from DWIET Engineering College, Punjab, India. She is having four years of teaching experience. She has one paper inDSP in Internationaljournal of comuter technology. Her areas of interestinclude Advanced Digital Signal Processing and VLSI

Dr. Rajesh Mehra: Dr. Mehra is currently associated with Electronics and Communication Engineering Department of National Institute of Technical Teachers'Training \& Research, Chandigarh, India since 1996. He has received his Doctor of Philosophy in Engineering and Technology from Panjab University, Chandigarh, India in 2015. Dr. Mehra received his
Master of Engineering from Panjab Univeristy, Chandigarh, India in 2008 and Bachelor of Technology from NIT, Jalandhar, India in 1994. Dr. Mehra has 20 years of academic and industry experience. He has more than 250 papers in his credit which are published in refereed International Journals and Conferences. Dr. Mehra has $55 \mathrm{ME}$ thesis in his credit. He has also authored one book on PLC \& SCADA. His research areas are Advanced Digital Signal Processing, VLSI Design, FPGA System Design, Embedded System Design, and Wireless \& Mobile Communication. Dr. Mehra is member of IEEE and ISTE. 